\title{
A Novel Discovery of Growth Process for Ag Nanowires and Plausible Mechanism
}

\author{
Jiejun Zhu, ${ }^{1,2}$ Caixia Kan, ${ }^{3}$ Yaozheng Wu, ${ }^{1}$ Jianguo Wan, ${ }^{1}$ Min Han, ${ }^{1}$ and Guanghou Wang ${ }^{1}$ \\ ${ }^{1}$ National Laboratory of Solid State Microstructures and Department of Physics, Nanjing University, Nanjing 210093, China \\ ${ }^{2}$ Department of Applied Physics, Nanjing University of Technology, Nanjing 210009, China \\ ${ }^{3}$ College of Science, Nanjing University of Aeronautics and Astronautics, Nanjing 211100, China \\ Correspondence should be addressed to Jianguo Wan; wanjg@nju.edu.cn
}

Received 12 June 2016; Accepted 31 August 2016

Academic Editor: Nathan C. Lindquist

Copyright (c) 2016 Jiejun Zhu et al. This is an open access article distributed under the Creative Commons Attribution License, which permits unrestricted use, distribution, and reproduction in any medium, provided the original work is properly cited.

A novel growth process of silver nanowires was revealed by tracing the morphology evolution of Ag nanostructures fabricated by an improved polyol process. A mixture of Ag nanowires and nanoparticles was obtained with the usage of PVP-K25 (MW = 38,000). The products sampled at different reaction time were studied in detail using UV-visible absorption spectra and transmission electron microscopy (TEM). An interesting phenomenon unknown in the past was observed where Ag nanoparticles undergo an important dissolution-recrystallization process and Ag nanowires are formed at the expense of the preformed Ag nanoparticles. A plausible novel growth mechanism for the silver nanowires was proposed.

\section{Introduction}

Noble metal nanostructures have been an attractive research subject due to their unique or improved electronic, catalytic, and optical properties. Compared with the isotropic spherical nanoparticles, anisotropic nanomaterials, especially metal nanowires, have attracted intensive interest because of their great potential for practical applications in nanoscale electronics, optoelectronics, and molecular sensing devices [1-5]. Recently, silver nanowires are of great interest because of their high electrical and thermal conductivities properties among all metals [6]. Our recent research indicated that silver nanowires, which are assembled with graphene to form a hybrid film, can drastically improve electrical conductivity of the polycrystalline graphene films [7].

Many approaches have been developed for the synthesis of high quality $\mathrm{Ag}$ nanowires with well-controlled shape and size to help research in studying their novel properties and applications. Among the synthetic approaches available, the common chemical reduction method is the capping agentmediated polyol process, in which poly(vinylpyrrolidone) (PVP) has been frequently used as a crucial reagent to control the growth rates of various facets of metal crystals [8-11]. In our previous work, it was found that both the concentration ratio $[\mathrm{PVP}] /\left[\mathrm{AgNO}_{3}\right]$ and chain length of PVP play important roles in synthesis of Ag nanowires by an improved polyol process [12]. By adjusting these two parameters precisely, the yields and aspect ratios of $\mathrm{Ag}$ nanowires could be controlled easily.

In the fabricating Ag nanowires with well-controlled dimensions and aspect ratios, it is necessary to understand the exact growth mechanism. In the past few decades, a common view is that the nanowires are grown from decahedral seed with assistance of PVP which interacted more strongly with $\mathrm{Ag}$ atoms on the $\{100\}$ facets than those on the $\{111\}$ facets. Therefore, with the presence of the PVP, the formed twinned nuclei with decahedral features can promote anisotropic growth along the fivefold axial [110] direction $[10,13]$. However, with more and further experiments carried out, the exact mechanism for the formation of $\mathrm{Ag}$ nanowires via this solution-phase approach is still not very clear. And the lateral growth behavior of fivefold-twinned nanowires has seldom been studied before (e.g., what is the lateral growth process?). Therefore, it is necessary to further investigate the morphological evolution of the Ag nanowires.

In our present work, depending on the careful investigation on the growth process of $\mathrm{Ag}$ nanowires in a polyol 
process, we discovered a novel and interesting lateral growth process through surface aggregation, which to the best of our knowledge is never reported ever before. This observation is significant not only for understanding the growth mechanism of Ag nanowires but also for the synthesis of metallic wires with tunable diameters which can be used for meeting various requirements in different practical applications.

In this paper, we focus on the morphological evolution of Ag nanowires. UV-visible absorption spectrometer, scanning electron microscopy (SEM), and transmission electron microscopy (TEM) were used to monitor the morphological evolution of the Ag nanostructures. And the possible twostep growth process for $\mathrm{Ag}$ nanowires was first proposed.

\section{Experiment Section}

$\mathrm{AgNO}_{3}$ ( $\geq 99.8 \%$, Sinopharm Chemical Reagent Co., Ltd.) and ethylene glycol (EG, Nanjing Chemical Reagent Co., Ltd.) were used in this work. PVP-K25 powder (Boai New Kaiyuan Pharmacy Co., Ltd.) with the average molecular weight (MW) of 38000 was chosen. All reagents were not purified further.

Ag nanostructures were synthesized by the improved PVP-mediated polyol process without any exotic seeds and ions. In our previous work, it has been demonstrated that the yields and aspect ratios of Ag nanowires could be controlled easily by adjusting the MW of PVP. Here, we choose PVP-K25 with average MW of 38000 as representative to synthesize Ag nanostructures. In this so-called polyol process, ethylene glycol served as both solvent and reducing agent. Firstly, $0.204 \mathrm{~g}$ of $\mathrm{AgNO}_{3}$ and $0.1332 \mathrm{~g}$ PVP were, respectively, solved in a $5 \mathrm{~mL}$ EG solvent. Then these two reactive solutions were simultaneously injected dropwise into $20 \mathrm{~mL}$ EG solvent which was magnetically stirred at $150^{\circ} \mathrm{C}$ in a round-bottom flask. The injection process lasted about $3 \mathrm{~min}$. Magnetic stirring was applied throughout the entire synthesis. The molar ratio between the repeating unit of PVP and $\mathrm{AgNO}_{3}$ was fixed at $R=1$.

The reactant mixture changes quickly from colorless to yellow after the addition of a few drops of PVP and $\mathrm{AgNO}_{3}$ solutions. The mixture turned from yellow to slightly opaque after $\sim 30$ min and finally formed slightly green colloids after $\sim 65 \mathrm{~min}$. For characterization, the mixture was sampled at different times and was then centrifuged repeatedly to remove possible contamination (EG and excess PVP).

For field-emission scanning electron microscopic (FESEM; Sirion 200, FEI, Holland) and X-ray diffraction (XRD; Ultima-III; Rigaku, Tokyo, Japan) measurements, the condensed products were dispersed on the copper sheets and glass substrates, respectively. For transmission electron microscopy (TEM; JEM-2000EX; JEOL) and high-resolution (HR) TEM (Tecnai G2 F20, FEI) observations, the products were diluted with deionized water $(\sim \times 30)$ and a few drops of dispersions were placed onto the carbon-coated copper grids and dried at room temperature. Optical absorption spectra for the diluted samples were recorded on a UV-vis-NIR spectrophotometer (U-3410, Hitachi). Fourier Transform Infrared (FT-IR) spectra were obtained on a Bruker Vector 22 FT-IR spectrophotometer by using $\mathrm{KBr}$ pellets.

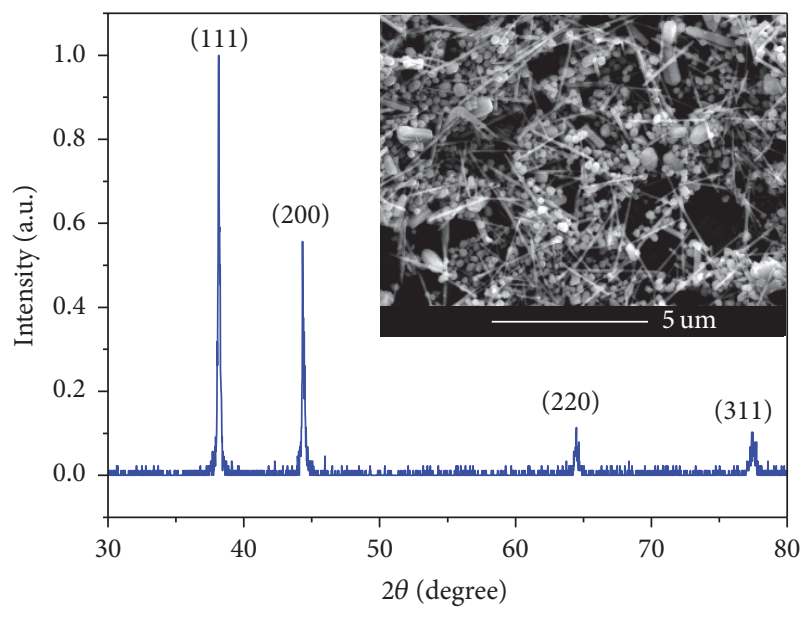

FIGURE 1: XRD results and the corresponding FE-SEM images of the final products synthesized with PVP-K25.

\section{Results and Discussion}

Figure 1 presents the XRD patterns and corresponding FESEM results of the final products. It can be seen that the Ag nanowires $(l=3 \mathrm{um}, d=100 \mathrm{~nm}$, and $r=l / d=$ 30) and a lot of irregular particles appear in the products (inserted in Figure 1). The XRD patterns indicate that Ag nanostructures synthesized using this solution-phase method are purely in the face-centre cube (fcc) phase. The lattice constant calculated from this XRD pattern was 4.092, which is perfectly in agreement with the literature value of 4.086 . We can also analyze the preferential orientation of crystals based on diffraction intensity of the XRD pattern. The ratio of intensity between the (111) and (200) peaks exhibits a relatively high value indicating the enrichment of the (111) crystalline planes in the as-synthesized $\mathrm{Ag}$ nanowires.

Theoretical calculations [14, 15] and experiments [16-18] demonstrate that the optical properties of metal nanoparticles depend on their shape, size, and composition. For example, the intensity and position of surface plasma resonance (SPR) of the Ag nanoparticles have a strong correlation with their exact morphology and the aspect ratio [19]. Recently, we calculated the optical resonances of Ag nanorods with fivefold-twinned structure based on the finite difference time domain (FDTD) solution; the results show that the weak longitudinal absorption peak of Ag nanorods located in near infrared radiation (NIR) and there are strong absorption peaks at about 350 and $390 \mathrm{~nm}$, when the aspect ratio is larger than 3 (see Figure 2). Since Ag nanostructures with different shapes and sizes exhibit different SPR bands at different frequencies, the morphological evolution of the samples was tracked by UV-vis-NIR spectrum measurements at various reaction times. In order to ensure in situ measurement, products were all measured after sampling at once and put into ice-cream water to prevent further increase of TEM measurement.

Figures 3(a) and 3(b) show the optical extinction spectra and the corresponding photos of Ag colloid solutions sampled at different times. The reactant mixture changes quickly 


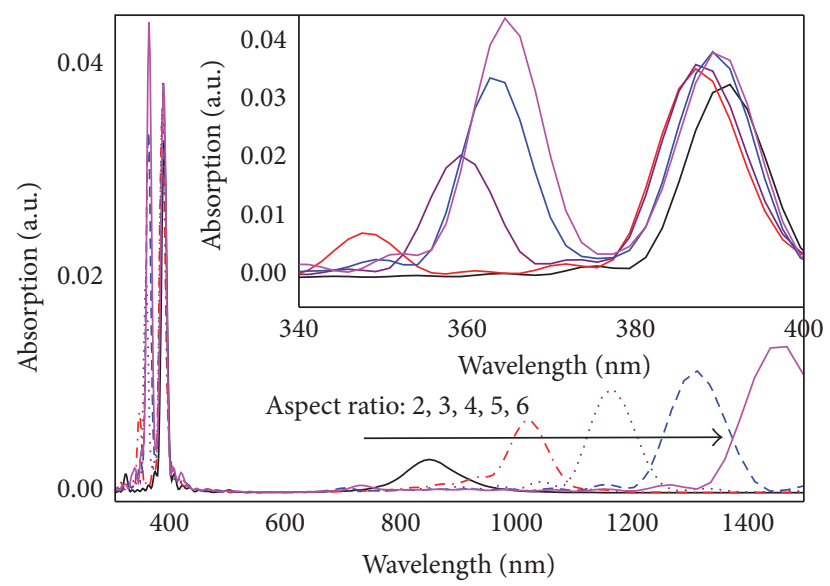

FIGURE 2: Simulated absorption spectra using finite difference time domain (FDTD) solution for Ag nanorods with fivefold-twinned structure. The inset is the magnified image.

from colorless to yellow after the addition of a few drops of PVP and $\mathrm{AgNO}_{3}$ solutions and a weak plasmon peak at $\sim 406 \mathrm{~nm}$ (curve A of Figure 3(a)) appears immediately, indicating the formation of $\mathrm{Ag}$ colloids with nanometresized dimensions and at a relatively low concentration. The mixtures turned from yellow to slightly opaque after $\sim 30 \mathrm{~min}$, and the peak mentioned above shifted to $\sim 430 \mathrm{~nm}$, indicating an increase in size for $\mathrm{Ag}$ nanoparticles (curve G of Figure 3(a)). After $\sim 65$ min slightly green colloids formed. As for the final products, there are three absorption bands $(\sim 355 \mathrm{~nm}$, $\sim 393 \mathrm{~nm}$, and $\sim 440 \mathrm{~nm}$ ) in the spectrum. From the measured optical absorption spectra and the FDTD simulations, it is also suggested that the final product should be a mixture of Ag nanowires and nanoparticles (curve $\mathrm{N}$ of Figure 3(b)). The corresponding spectra of the final products are in agreement with the SEM results (see inset in Figure 1).

In the optical spectra, as the reaction proceeded to $32 \mathrm{~min}$, a new absorption shoulder appears at $\sim 600 \mathrm{~nm}$ (curve $\mathrm{H}$ of Figure 3(b)). This absorption implies the formation of Ag nanorods with small aspect ratios, whose longitudinal plasmon resonance could contribute to the appearance of absorption at long wavelengths. As the Ag nanorods grew with time, the transverse plasmon mode of nanowires (at about $380 \mathrm{~nm}$ ) was observed at $t=37 \mathrm{~min}$, while the longitudinal plasmon resonance essentially disappeared (curve I of Figure 3(b)). During this period (30-39 min), the peak positioned at $\sim 425 \mathrm{~nm}$ broadened and shifted to $\sim 450 \mathrm{~nm}$ (curves G-J of Figure 3(b)), indicating an increase in size of $\mathrm{Ag}$ nanoparticles. As the reaction proceeded from 39 to 50 min (curves J-L of Figure 3(b)), two plasmon peaks at 380 and $350 \mathrm{~nm}$ were further increased in intensity, while the plasmon resonance of $\mathrm{Ag}$ nanoparticles at $\sim 448 \mathrm{~nm}$ decreased quickly, indicating the decrease (in size and number) of Ag nanoparticles. It is worth noting that, during a very short period from 50 to $55 \mathrm{~min}$, a red shift from $\sim 380 \mathrm{~nm}$ to $393 \mathrm{~nm}$ (curves L-M of Figure 3(b)) of the Ag nanowires implies that the diameter of the $\mathrm{Ag}$ nanowires becomes larger. At the same time, the absorption of Ag nanoparticles at $\sim 440 \mathrm{~nm}$ appeared again (accompanied with absorption of Ag nanowires). Therefore, the optical spectra indicated that the final product synthesized with the usage of PVP-K25 was a mixture of $\mathrm{Ag}$ nanowires and nanoparticles.

For direct observation of the morphological evolution of Ag nanostructures, TEM was also used to characterize the samples at different stages. And the changes in these images correlate well with the optical features (Figures 3(a) and $3(\mathrm{~b})$ ). Figures $4(\mathrm{a})-4(\mathrm{~g})$ give typical TEM images of the products at $30,32,39,45,50,55$, and $65 \mathrm{~min}$ after the addition of PVP and $\mathrm{AgNO}_{3}$ solutions. The images clearly present the growth process of $\mathrm{Ag}$ nanostructures. In the period from $30 \mathrm{~min}$ to $39 \mathrm{~min}$, it can be seen that the length of Ag nanowires increased from $\sim 100 \mathrm{~nm}$ to several microns. However, the average diameter of the Ag nanowires was about $30 \mathrm{~nm}$ without obvious change during this course of the observation. In the same period, the size of Ag nanoparticle was increased from $30 \mathrm{~nm}$ to about $60 \mathrm{~nm}$ (see Figures $4(\mathrm{a})-4(\mathrm{c})$ ). When the reaction proceeded to $45 \mathrm{~min}$, many nanoparticles attached to the Ag nanowires like sugar-coated haws, and some of them dissolved into smaller particles (surface of the particles is rough), as shown in Figure 4(d). After another five minutes, there were no monodisperse $\mathrm{Ag}$ nanoparticles and almost all nanoparticles assembled together with Ag nanowires uniformly (as seen in Figures 4(e1) and 4(e2)). By observation of the right magnified images (Figure 4(e2)), these nanoparticles attached on the Ag nanowires have been dissolved to smaller nanoparticles. This result is in accordance with the UV-vis-NIR spectrum measurements at the same time (curve L in Figure 3(b)), which indicated that the plasmon resonance of nanoparticles at $\sim 448 \mathrm{~nm}$ decreased quickly. The SEM result also indicated that most of the Ag nanowires were decorated with nanoparticles and the side surfaces became rough with attachment of the dissolved nanoparticles (see SEM result in Figure 5). The energy dispersive spectroscopy (EDS) with $\mathrm{Cu}$ slide as a substrate confirmed the chemical composition of these nanoparticles attached on the Ag nanowires was only silver element (as seen in Figure 5). Subsequently, during the period from $50 \mathrm{~min}$ to $65 \mathrm{~min}, \mathrm{Ag}$ nanowires with lager average diameter $(\sim 100 \mathrm{~nm})$ and irregular particles appeared in the solution (see Figures 4(f) and 4(g)). The average length of Ag nanowires was about several microns and remained almost unchanged after about $40 \mathrm{~min}$, noting that there were lots of very small nanoparticles attached on the surface of the Ag nanowires (see the magnified images of Figures 4(f) and $4(\mathrm{~g}))$.

According to the above observations, it is proposed that the formed Ag nanoparticles after $\sim 40$ min underwent the following three processes: assembly, oriented by $\mathrm{Ag}$ nanowires, dissolution, and recrystallization. It is worth noting that this is recrystallization rather than an aggregation process. On the basis of the selected-area electron diffraction (SAED) and high-resolution transmission electron microscopy (HRTEM) studies, the as-synthesized Ag nanowires showed high crystallinity. Figure 6 shows the TEM image of final products after centrifugation. Inset 1 gives the magnified images of the end of one Ag nanowire (arrowed in Figure 6). Insets 2 and 3 show the SAED and HRTEM of 


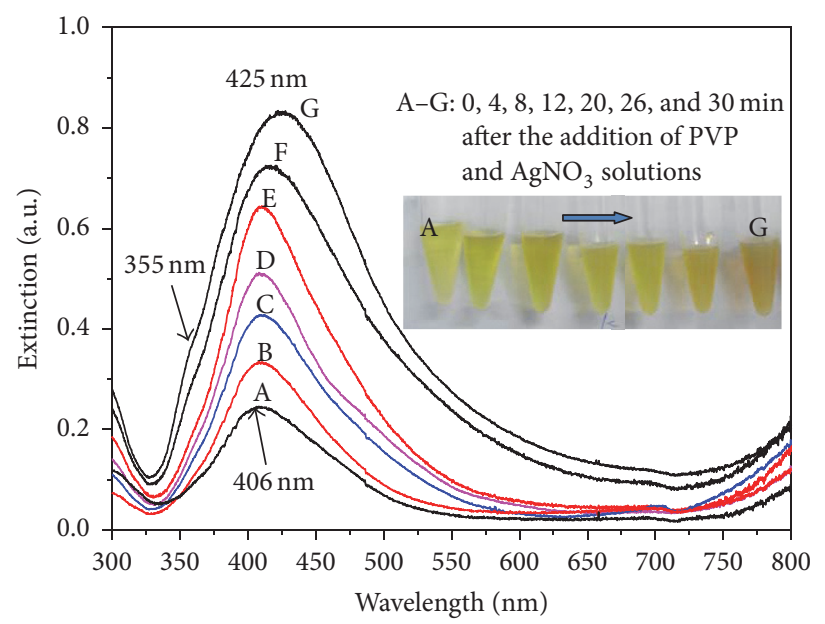

(a)

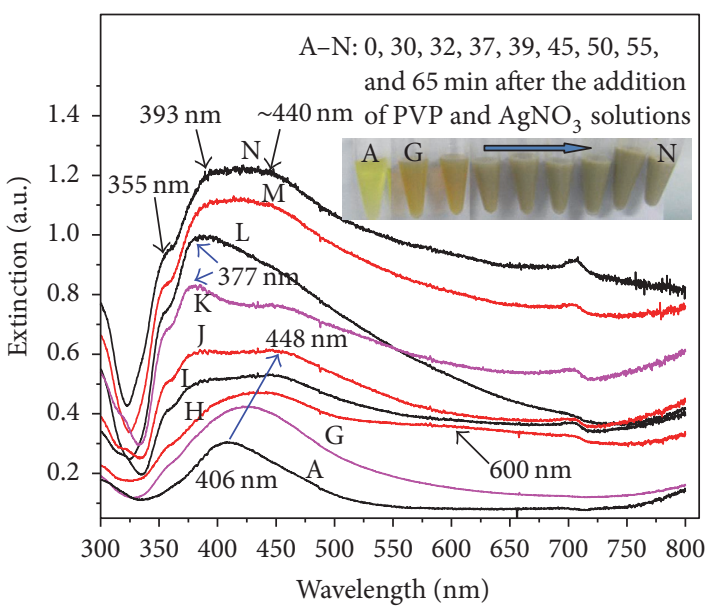

(b)

FIgURE 3: The optical extinction spectra and the corresponding photos of Ag colloid solutions sampled at different time.

the Ag nanowire in inset 1, respectively. The SAED pattern consists of a superimposition of contributions from different subunits. The spots of superimposition from $\left[\begin{array}{lll}1 & 1 & 0\end{array}\right]$ zone axis of units T2 and T5 are indicated. According to the HRTEM image, the fringe spacing is about $0.235 \mathrm{~nm}$, which is in agreement with the (111) lattice spacing of Ag crystal (see inset 3 of Figure 6). There is $18^{\circ}$ rotation around the $O O^{\prime}$ central axis of the Ag nanowire comparing the substrate. The results of Figure 6 imply that the Ag nanowires synthesized in this condition have fivefold symmetry, as widely reported about Ag nanowires [20-22].

\section{Crystal Growth Mechanism}

Now we present our understanding of the formation mechanism of the Ag nanowires.

For the process of longitudinal growth of Ag nanorods, it is usually believed that the regular decahedron seeds could be "elongated" to pentagonal wires/rods based on two possible growth mechanisms. One is strain-restriction mechanism $[23,24]$. The defect energy that exists along the (111) twinning planes between five crystal units limits the lateral growth as it would significantly increase the faulty area and hence the energy in the system. In contrast, the growth along the longitudinal axis of rod causes a relatively smaller increment of the faulty area and this growth is favored. However, this strain-restriction mechanism is not sufficient to induce this anisotropic growth. For example, Zhang et al. proposed that the reentrant grooves should release the internal strain effectively [25]. Another explanation proposed that PVP interacted more strongly with Ag atoms on the 200 facets than those on the $\left\{\begin{array}{lll}1 & 1 & 1\end{array}\right\}$ facets [10], which obviously slows (or prevents) the growth on the (111) plane and promotes a highly anisotropic crystal growth along the longitudinal axis. However, there is a question that the regular decahedral seeds have no (200) facets for the adsorption of surface-capping agents. So, the actual mechanism of the elongation process is still under debate.
For our cases, the TEM observations and optical spectra provide new information for the growth mechanism of $1 \mathrm{D}$ Ag nanostructures. According to the obtained results, we proposed that there are two major steps in the growth process of Ag nanowires. The first step is the growth along the longitudinal axis due to the role of PVP. The second novel step is the growth along the lateral axis, during which an interesting phenomenon unknown in the past was observed where Ag nanoparticles undergo an assembly, dissolution, and recrystallization process. A proposed schematic illustration for the growth process of $\mathrm{Ag}$ nanowires is shown in Figure 7.

At the first step of synthesis process, PVP plays a very important role. We know that PVP has the structure of polyvinyl skeleton with strong polar group (pyrrolidone ring) and has an affinity toward many chemicals to form coordinative compounds. The polar groups, such as the $>\mathrm{C}=\mathrm{O}$ groups of $\mathrm{PVP}$ chain, can interact with metal ions and form coordinating complex [26]. In our previous work [27], Fourier Transform Infrared spectra of PVP and PVP$\mathrm{AgNO}_{3}$ film were measured. And a shift in frequency of the $\mathrm{C}=\mathrm{O}$ in pyrrolidone ring indicates an interaction between the $\mathrm{AgNO}_{3}$ molecules and the PVP chains. So, we propose that the coordination compound of $\mathrm{Ag}^{+}$and the PVP should be formed accompanying those free $\mathrm{Ag}^{+}$at the initial stage (like Figure 7(a)). These reactions would create a state in which $\mathrm{Ag}^{+}$ions can be reduced to $\mathrm{Ag}^{0}$ to form extremely fine $\mathrm{Ag}$ embryos with $1 \mathrm{D}$ structure. At the same time, the $\mathrm{Ag}^{+}$ions are reduced to $\mathrm{Ag}$ and then nucleate or grow on the $\mathrm{Ag}$ embryos rapidly (like Figure $7(\mathrm{~b})$ ) due to the following reactions at high temperatures:

$$
\begin{aligned}
2 \mathrm{HOCH}_{2} \mathrm{CH}_{2} \mathrm{OH} \longrightarrow & 2 \mathrm{CH}_{3} \mathrm{CHO}+2 \mathrm{H}_{2} \mathrm{O} \\
2 \mathrm{Ag}^{+}+2 \mathrm{CH}_{3} \mathrm{CHO} \longrightarrow & \mathrm{CH}_{3} \mathrm{CO}-\mathrm{OCCH}_{3}+2 \mathrm{Ag} \\
& +2 \mathrm{H}^{+}
\end{aligned}
$$




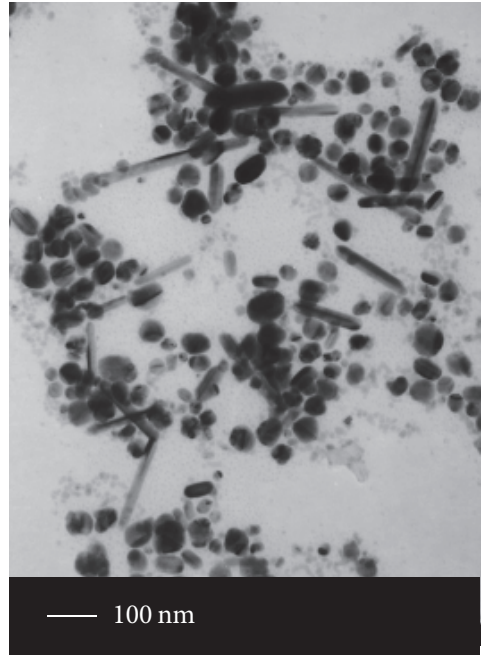

(a)

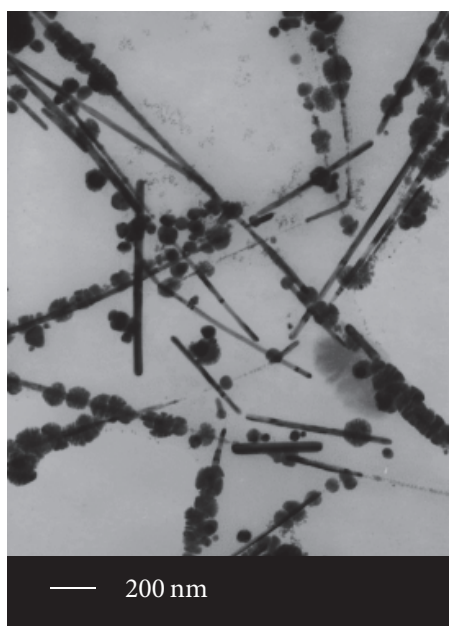

(d)
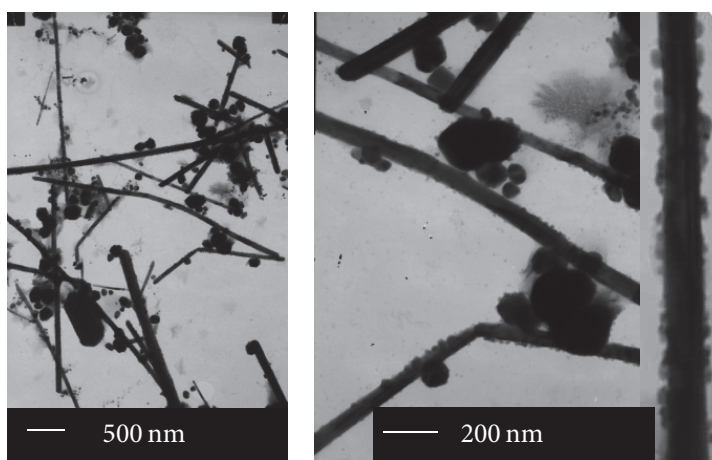

(f)

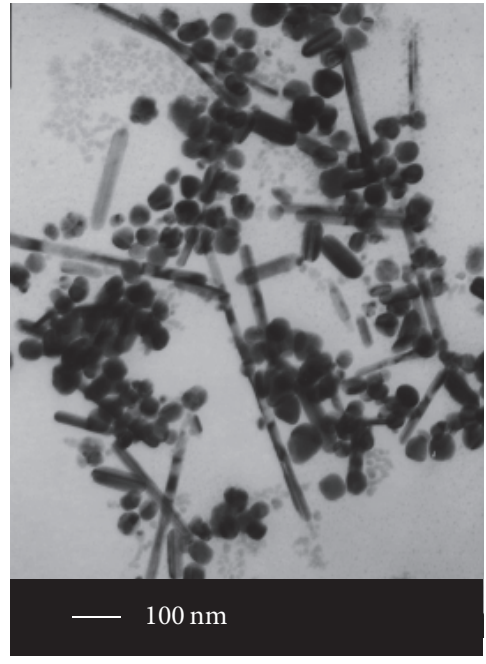

(b)

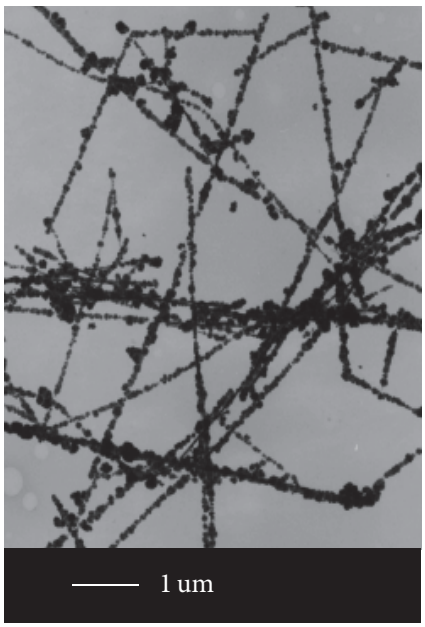

(e1)

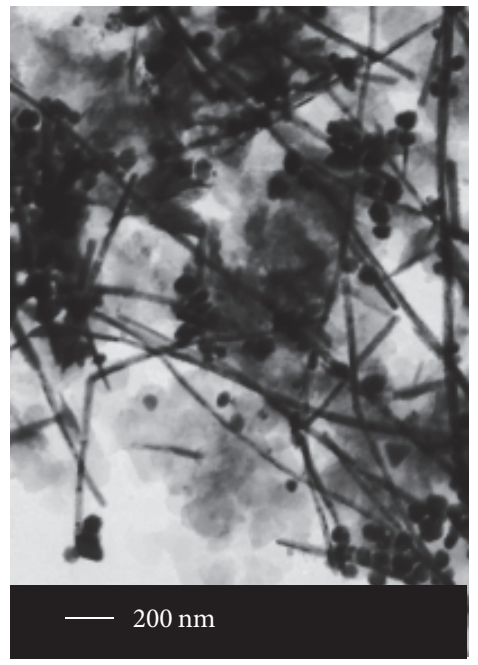

(c)

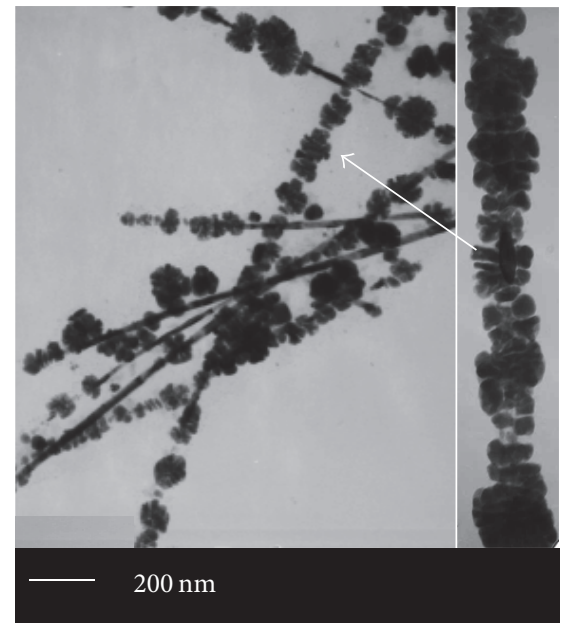

(e2)
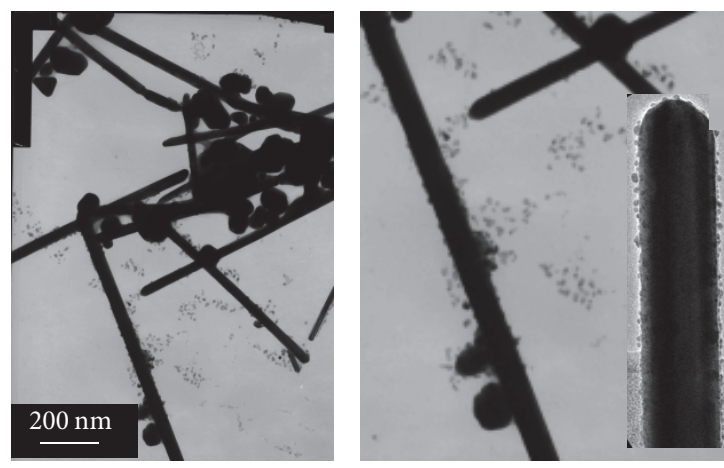

(g)

FIGURE 4: TEM images of Ag nanostructures synthesized with the usage of PVP-K25 and sampled at (a) $30 \mathrm{~min}$, (b) $32 \mathrm{~min}$, (c) 39 min, (d) $45 \mathrm{~min}$, (e1, e2) $50 \mathrm{~min}$, (f) $55 \mathrm{~min}$, and (g) $65 \mathrm{~min}$ after the addition of PVP and $\mathrm{AgNO}_{3}$ solutions, corresponding to the samples of curves in Figure 3(b). 

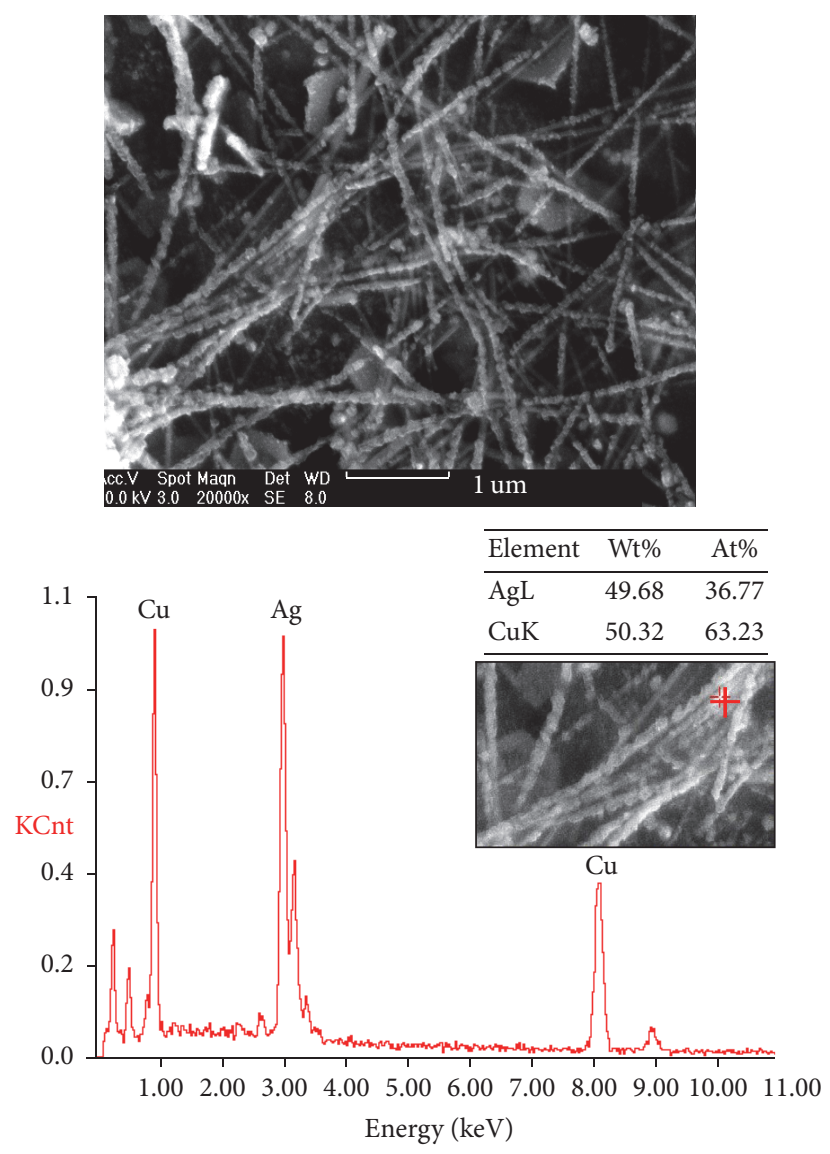

FIGURE 5: FE-SEM image and EDS pattern of the product corresponding to the sample of Figures 4(e1) and 4(e2).

PVP has another important role acting as a growthdirecting reagent and the growth rates of crystal are often controlled (kinetically rather than thermodynamically) by the sticking probability on a given face [28]. In the study of the growth mechanism of anisotropic Ag nanostructures fabricated by the polyol process, it is verified that PVP interacted more strongly with Ag atoms on the $\{100\}$ facets than those on the $\left\{\begin{array}{lll}1 & 1 & 1\end{array}\right\}$ facets [10], which obviously slows (or prevents) the growth on the (111) plane and promotes a highly anisotropic crystal growth along the longitudinal axis. Therefore, with the presence of the growth-directing reagent of PVP, the formed Ag nanowires can promote preferential growth along the longitudinal axis and the length would be enlarged (like Figure 7(c)). At the same time, the Ag nanoparticles will be formed due to nucleation and the attachment of the free Ag atoms. Thus the PVP-Ag coordination complex compound would promote the formation of $\mathrm{Ag}$ nanowires in hot EG solution. Figures $7(\mathrm{a})-7(\mathrm{c})$ demonstrate the process of coordination, nucleation, and growth (grow longer or larger for $\mathrm{Ag}$ nanowires and nanoparticles, resp.), which corresponds to the period of $0 \sim 40 \mathrm{~min}$.

The subsequent process is the lateral growth of $\mathrm{Ag}$ nanowires. The lateral growth behavior of fivefold-twinned $\mathrm{Ag}$ nanowires is an interesting phenomenon. To the best of our knowledge, this phenomenon was never reported in the

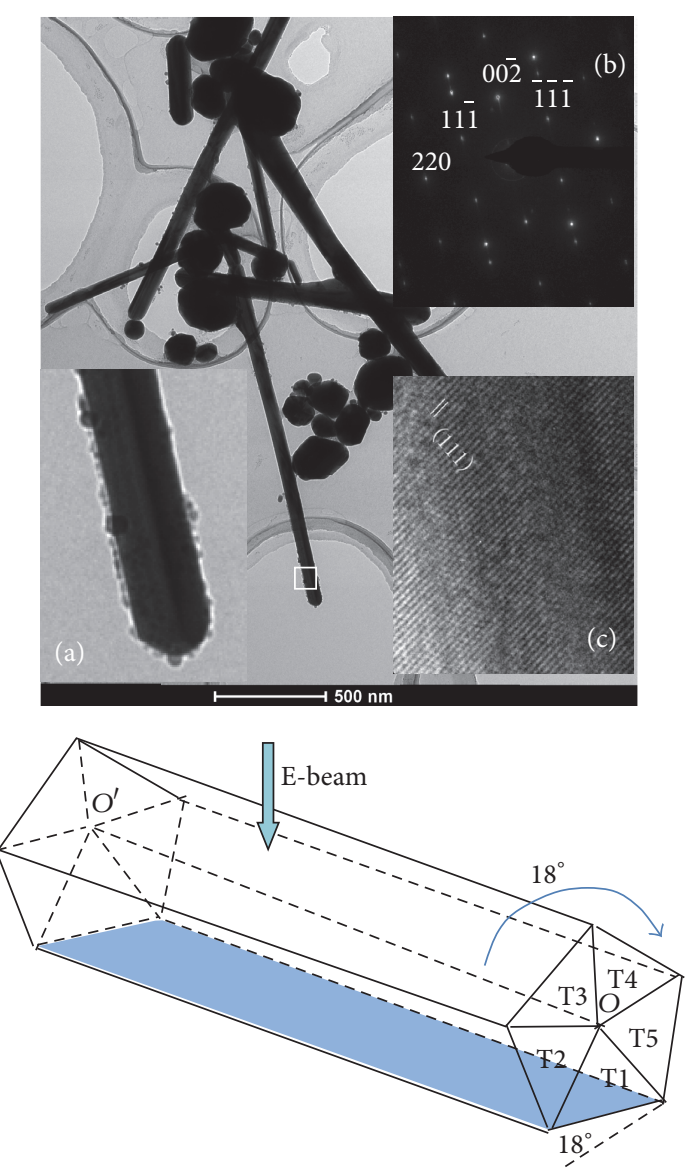

FIGURE 6: TEM image of the final Ag nanoproducts using PVP-K25. Inset (a): the nanowire used for the SAED and HRTEM studies with the area marked with a white pane indicating where the HRTEM image was recorded. Inset (b): a typical SAED of one Ag nanowire (following diagram is the orientation of the nanowires with respect to the incident beams). The spots from superimposition of the [110] axis are indicated. Inset(c): a typical HRTEM image of a selected area of an individual Ag nanowire.

past. In the previous literatures, it is generally considered that the lateral growth was always restricted for the strainrestriction and the growth-directing mechanism of PVP. However, in our experiment, the lateral sizes become lager during a very short period (about $15 \mathrm{~min}$ ). According to the TEM observation during the time from $39 \mathrm{~min}$ to $55 \mathrm{~min}$, it is suggested that the $\mathrm{Ag}$ nanoparticles formed in the first step undergo an important assembly, dissolution, and recrystallization process and act as fuel for the lateral growth of nanowires. In general, crystal growth is always both thermodynamically and kinetically controlled. It is proposed that the nanoparticles would be assembled near the as-formed nanowires (like Figure $7(\mathrm{~d})$ ) due to the continuous collision with the Ag nanowires in the hot reaction system. And then many Ag nanoparticles begin to dissolve (like Figure 7(e)) and act as fuel for the lateral growth of $\mathrm{Ag}$ nanowires in order to attain a lower energy state (thermodynamically favored), which is similar to the process of Ostwald Ripening (like Figure 7(f)). There are some nanoparticles in the final 


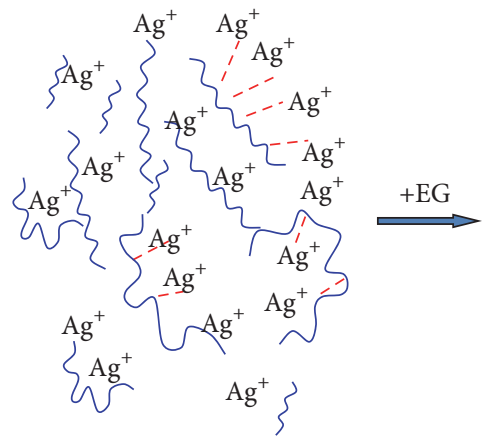

(a)

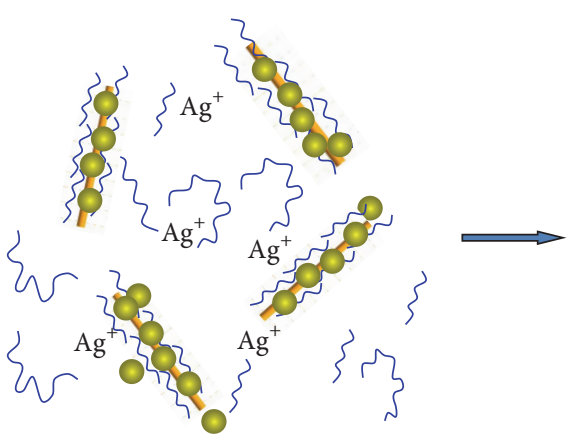

(d)

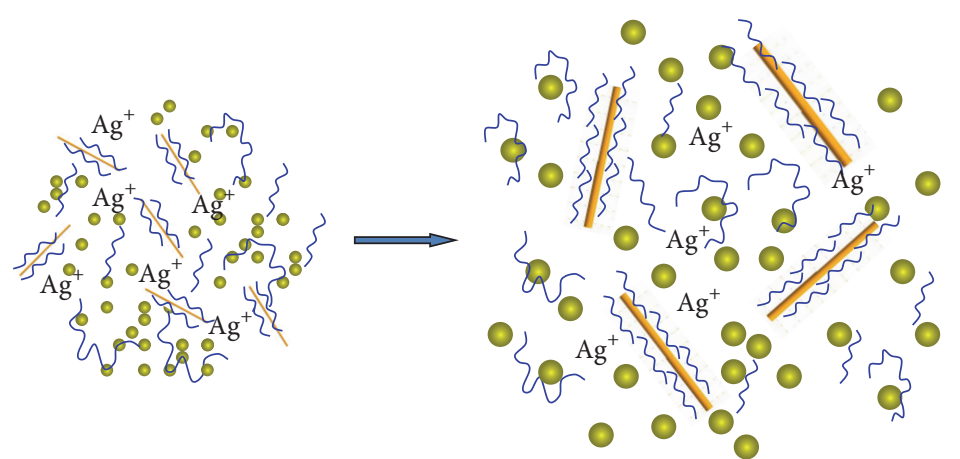

(b)

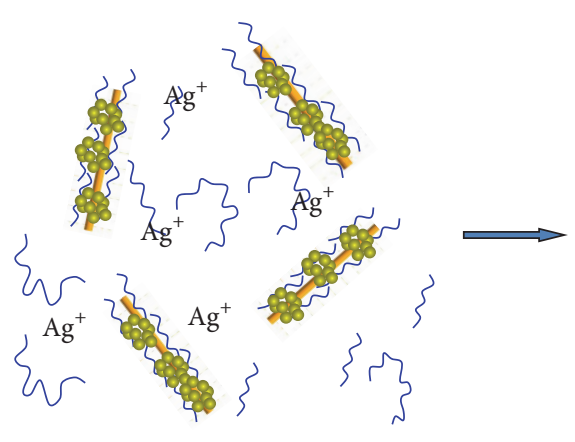

(e) (c)

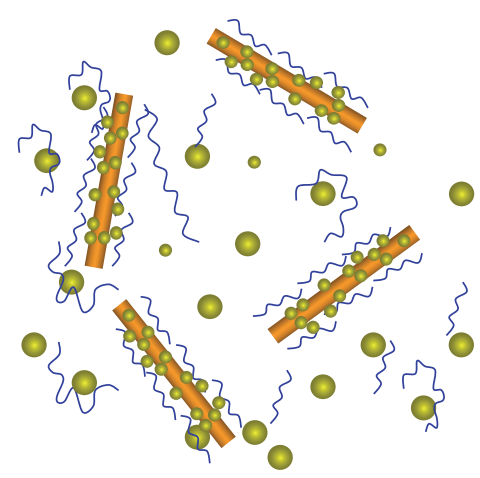

(f)

FIGURE 7: Schematic illustrations of proposed growth mechanisms of the synthesized silver nanowires. (a) Complexation of Ag ions with PVP chains. (b) Nucleation for 1D structures and particles. (c) Formation of silver nanowires and nanoparticle. (d) Nanoparticles assembly directed by the nanowires. (e) Dissolution of silver nanoparticles. (f) Formation of thicker silver nanowires at the expense of the silver nanoparticle (silver nanoparticles appear again due to the secondary nucleation and growth).

products probably because of the secondary nucleation and growth by the reduction of excess $\mathrm{Ag}^{+}$.

\section{Conclusion}

In summary, $\mathrm{Ag}$ nanowires and nanoparticles were synthesized with the usage of PVP-K25 by an improved polyol process. According to the investigations in detail through the UV-visible absorption spectra and TEM, an interesting phenomenon unknown in the past was observed where Ag nanoparticles undergo an important dissolutionrecrystallization process and act as source for the lateral growth of nanowires. And two possible steps of growth process for Ag nanowires were first proposed. One is the growth along longitudinal axis through the coordination and direction of PVP which is kinetically controlled. The second novel step is the growth along the lateral axis at the expense of the Ag nanoparticles, which is thermodynamically controlled. Although the exact reason why the Ag nanoparticles assembled near the nanowires is not very clear, this work represents a significant step to gain a better understanding for the anisotropic growth of noble nanostructures and provides a method for the synthesis of metallic wires with tunable diameters which can be used for meeting various requirements in different practical applications.

\section{Competing Interests}

The authors declare that there is no conflict of interests regarding the publication of this paper.

\section{Acknowledgments}

This work was supported by the National Key Projects for Basic Research of China (Grant no. 2015CB921203), the National Natural Science Foundation of China (Grant nos. 51472113 and 11134005), and the PAPD project. The authors thank Dr. Zhaosheng Li for his great help with X-ray diffraction (XRD) measurements in his research group.

\section{References}

[1] Y. Xia, P. Yang, Y. Sun et al., "One-dimensional nanostructures: synthesis, characterization, and applications," Advanced Materials, vol. 15, no. 5, pp. 353-389, 2003.

[2] W. L. Barnes, A. Dereux, and T. W. Ebbesen, "Surface plasmon subwavelength optics," Nature, vol. 424, no. 6950, pp. 824-830, 2003.

[3] J. N. Anker, W. P. Hall, O. Lyandres, N. C. Shah, J. Zhao, and R. P. Van Duyne, "Biosensing with plasmonic nanosensors," Nature Materials, vol. 7, no. 6, pp. 442-453, 2008. 
[4] R. Wang and H. Ruan, "Synthesis of copper nanowires and its application to flexible transparent electrode," Journal of Alloys and Compounds, vol. 656, pp. 936-943, 2016.

[5] B. Li, S. Ye, I. E. Stewart, S. Alvarez, and B. J. Wiley, "Synthesis and purification of silver nanowires to make conducting films with a transmittance of 99\%," Nano Letters, vol. 15, no. 10, pp. 6722-6726, 2015.

[6] Z. Wang, J. Liu, X. Chen, J. Wan, and Y. Qian, "A simple hydrothermal route to large-scale synthesis of uniform silver nanowires," Chemistry, vol. 11, no. 1, pp. 160-163, 2005.

[7] H. B. Sun, G. X. Ge, J. J. Zhu et al., "High electrical conductivity of graphene-based transparent conductive films with silver nanocomposites," RSC Advances, vol. 5, no. 130, pp. 108044108049, 2015.

[8] Y. Sun and Y. Xia, "Large-scale synthesis of uniform silver nanowires through a soft, self-seeding, polyol process," Advanced Materials, vol. 14, no. 11, pp. 833-837, 2002.

[9] M. Tsuji, Y. Nishizawa, K. Matsumoto, M. Kubokawa, N. Miyamae, and T. Tsuji, "Effects of chain length of polyvinylpyrrolidone for the synthesis of silver nanostructures by a microwavepolyol method," Materials Letters, vol. 60, no. 6, pp. 834-838, 2006.

[10] Y. Sun, B. Mayers, T. Herricks, and Y. Xia, "Polyol synthesis of uniform silver nanowires: a plausible growth mechanism and the supporting evidence," Nano Letters, vol. 3, no. 7, pp. 955960, 2003.

[11] K. K. Caswell, C. M. Bender, and C. J. Murphy, "Seedless, surfactantless wet chemical synthesis of silver nanowires," Nano Letters, vol. 3, no. 5, pp. 667-669, 2003.

[12] J. J. Zhu, C. X. Kan, J. G. Wan, M. Han, and G. H. Wang, "High-yield synthesis of uniform ag nanowires with high aspect ratios by introducing the long-chain PVP in an improved polyol process," Journal of Nanomaterials, vol. 2011, Article ID 982547, 7 pages, 2011.

[13] Y. Gao, P. Jiang, L. Song et al., "Growth mechanism of silver nanowires synthesized by polyvinylpyrrolidone-assisted polyol reduction," Journal of Physics D: Applied Physics, vol. 38, no. 7, pp. 1061-1067, 2005.

[14] I. O. Sosa, C. Noguez, and R. G. Barrera, "Optical properties of metal nanoparticles with arbitrary shapes," Journal of Physical Chemistry B, vol. 107, no. 26, pp. 6269-6275, 2003.

[15] B. J. Wiley, Y. Chen, J. M. McLellan et al., "Synthesis and optical properties of silver nanobars and nanorice," Nano Letters, vol. 7, no. 4, pp. 1032-1036, 2007.

[16] J. P. Kottmann, O. J. F. Martin, D. R. Smith, and S. Schultz, "Plasmon resonances of silver nanowires with a nonregular cross section," Physical Review B, vol. 64, no. 23, Article ID 235402, 2001.

[17] J. P. Kottmann, O. J. F. Martin, D. R. Smith, and S. Schultz, "Dramatic localized electromagnetic enhancement in plasmon resonant nanowires," Chemical Physics Letters, vol. 341, no. 1-2, pp. 1-6, 2001.

[18] W.-H. Yang, G. C. Schatz, and R. P. Van Duyne, "Discrete dipole approximation for calculating extinction and Raman intensities for small particles with arbitrary shapes," Journal of Chemical Physics, vol. 103, no. 3, pp. 869-875, 1995.

[19] R.-L. Zong, J. Zhou, Q. Li et al., "Synthesis and optical properties of silver nanowire arrays embedded in anodic alumina membrane," Journal of Physical Chemistry B, vol. 108, no. 43, pp. 16713-16716, 2004.
[20] H. Hofmeister, S. A. Nepijkob, D. N. Ievlevb, W. Schulzeb, and G. Ertl, "Composition and lattice structure of fivefold twinned nanorods of silver," Journal of Crystal Growth, vol. 234, no. 4, pp. 773-781, 2002.

[21] H. Chen, Y. Gao, H. Yu et al., "Structural properties of silver nanorods with fivefold symmetry," Micron, vol. 35, no. 6, pp. 469-474, 2004.

[22] C. Ni, P. A. Hassan, and E. W. Kaler, "Structural characteristics and growth of pentagonal silver nanorods prepared by a surfactant method," Langmuir, vol. 21, no. 8, pp. 3334-3337, 2005.

[23] Y. Xiong and Y. Xia, "Shape-controlled synthesis of metal nanostructures: the case of palladium," Advanced Materials, vol. 19, no. 20, pp. 3385-3391, 2007.

[24] C. Lofton and W. Sigmund, "Mechanisms controlling crystal habits of gold and silver colloids," Advanced Functional Materials, vol. 15, no. 7, pp. 1197-1208, 2005.

[25] W. Zhang, Y. Liu, R. Cao et al., "Synergy between crystal strain and surface energy in morphological evolution of fivefold-twinned silver crystals," Journal of the American Chemical Society, vol. 130, no. 46, pp. 15581-15588, 2008.

[26] P. Jiang, S.-Y. Li, S.-S. Xie, Y. Gao, and L. Song, "Machinable long PVP-stabilized silver nanowires," Chemistry, vol. 10, no. 19, pp. 4817-4821, 2004.

[27] C. Kan, W. Cai, C. Li, and L. Zhang, "Optical studies of polyvinylpyrrolidone reduction effect on free and complex metal ions," Journal of Materials Research, vol. 20, no. 2, pp. 320324, 2005.

[28] L. D. Marks, "Experimental studies of small particle structures," Reports on Progress in Physics, vol. 57, no. 6, pp. 603-649, 1994. 

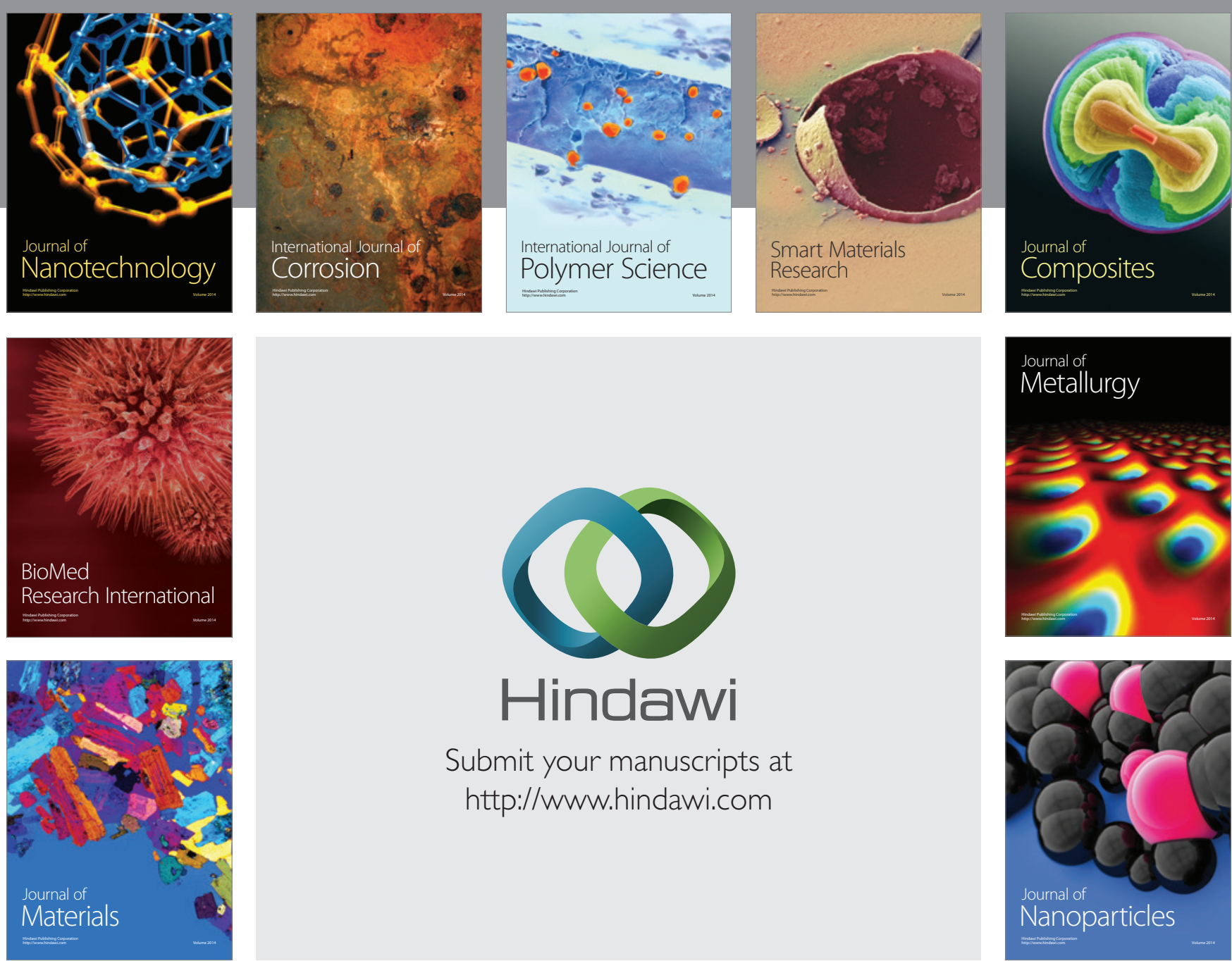

\section{Hindawi}

Submit your manuscripts at

http://www.hindawi.com

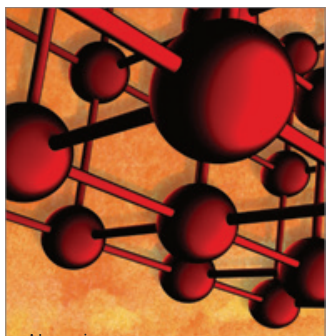

Materials Science and Engineering
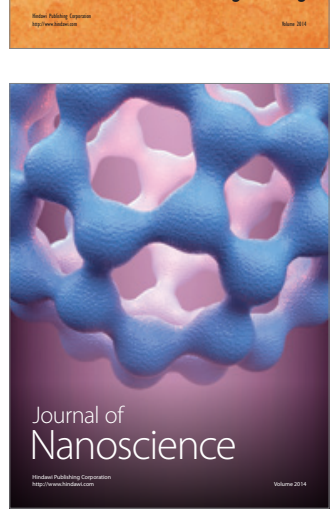
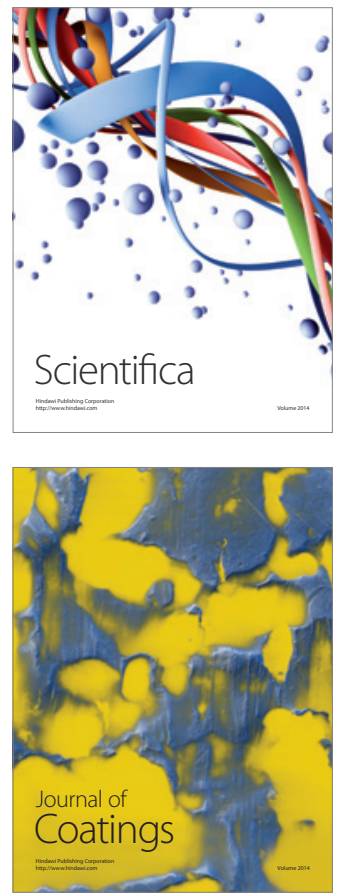
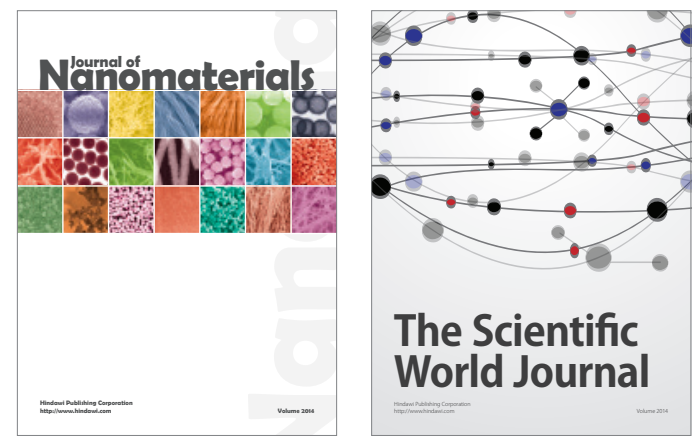

The Scientific World Journal
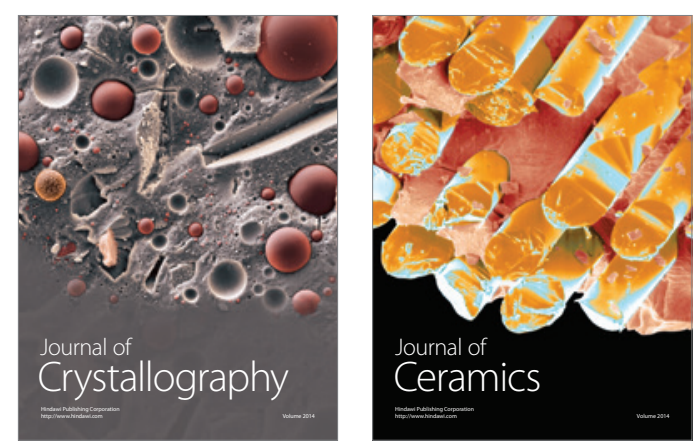
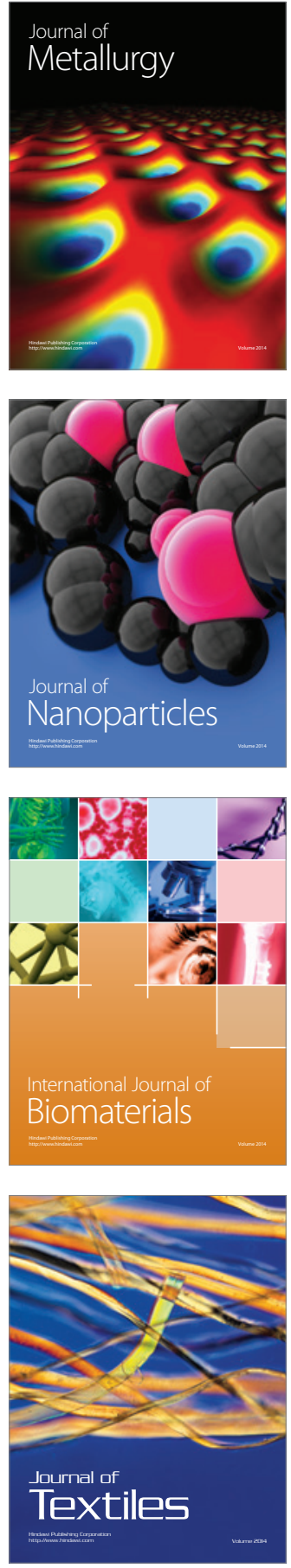